Discrete Comput Geom 32:117-128 (2004)

DOI: $10.1007 / \mathrm{s} 00454-003-2919-4$

\title{
Construction of a 3/4-Ideal Hyperbolic Tetrahedron out of Ideal Tetrahedra
}

\author{
Yana Mohanty \\ Department of Mathematics, University of California at San Diego, \\ La Jolla, CA 92093-0112, USA \\ mohanty@math.ucsd.edu
}

\begin{abstract}
We show how to construct an arbitrary 3/4-ideal hyperbolic tetrahedron out of 10 ideal tetrahedra.
\end{abstract}

\section{Introduction}

The study of hyperbolic simplices has gained interest in recent years due to its importance in the theory of hyperbolic 3-manifolds. Every hyperbolic manifold can be obtained by identifying the faces of a hyperbolic polyhedron, and the volume of such a manifold is a topological invariant by Mostow rigidity. It is therefore natural to study triangulations of hyperbolic 3-manifolds and the simplices which they produce.

In general, these triangulations consist of finite hyperbolic simplices which are characterized by six parameters. It would be more convenient to construct the finite tetrahedra out of ideal tetrahedra, each of which is determined by just two parameters. Such a construction is an immediate consequence of the result presented in this paper.

In seeking this construction, it is tempting to look at one of the volume formulas for a finite hyperbolic tetrahedron. This is because in hyperbolic space the volume of simplices and their decompositions are closely related, as can be seen in Milnor's derivation of the volume of an ideal hyperbolic tetrahedron in [5]. The volume is given by the famous formula

$$
V(T(\alpha, \beta, \gamma))=Л(\alpha)+Л(\beta)+Л(\gamma),
$$

where $T(\alpha, \beta, \gamma)$ is an ideal tetrahedron with dihedral angles $\alpha, \beta$, and $\gamma$ at each of its vertices, and $Л(\theta)=-\int_{0}^{\theta} \log 2|\sin u| d u$ is the Lobachevsky function. The three terms on the right-hand side of (1) represent the volumes of tetrahedra $\mathcal{L}(\alpha), \mathcal{L}(\beta)$, and $\mathcal{L}(\gamma)$, defined in Fig. 1. While several formulas for the volume of a finite hyperbolic tetrahedron 


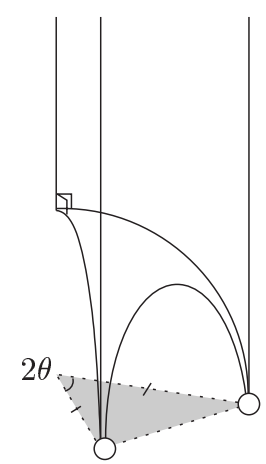

Fig. 1. Bilaterally symmetric $3 / 4$-ideal tetrahedron $\mathcal{L}(\theta)$ in the half-space model. The shaded isosceles triangle is the shadow cast by the tetrahedron onto the plane at infinity. The circles represent vertices at infinity.

in terms of the Lobachevsky function have recently appeared in the literature (see [2] or [7], [1], [8], and [6]), none of them are bona fide decompositions into tetrahedra of the type $\mathcal{L}(\theta)$. The main obstacle lies in obtaining a volume formula that is also a decomposition for a 3/4-ideal tetrahedron.

A well-known formula for the volume of such a tetrahedron, as well as its derivation, can be found in Section 5.2 of [11]:

$$
\begin{aligned}
V\left(T_{3}(A, B, C)\right)=\frac{1}{2}[ & Л(A)+Л\left(A^{\prime}\right)+Л(B)+Л\left(B^{\prime}\right)+Л(C)+Л\left(C^{\prime}\right) \\
& \left.-Л\left(\frac{\pi+A+B+C}{2}\right)\right],
\end{aligned}
$$

where $T_{3}(A, B, C)$ is a $3 / 4$-ideal tetrahedron with dihedral angles $A, B, C$ at its finite vertex and

$$
A^{\prime}=\frac{\pi+A-B-C}{2}, \quad B^{\prime}=\frac{\pi+B-A-C}{2}, \quad C^{\prime}=\frac{\pi+C-A-B}{2} .
$$

While it is clear from Fig. 1 that $Л(\theta) / 2$ represents the volume of one of the symmetric halves of $\mathcal{L}(\theta)$, it is far from obvious how to construct $T_{3}(A, B, C)$ from such objects.

Any finite tetrahedron can be constructed from an ideal tetrahedron and three 3/4-ideal tetrahedra as shown in Fig. 2. Since every ideal tetrahedron with dihedral angles $\alpha, \beta, \gamma$ decomposes into $\mathcal{L}(\alpha), \mathcal{L}(\beta)$, and $\mathcal{L}(\gamma)$, as noted earlier, the key step in constructing a finite hyperbolic tetrahedron out of tetrahedra of the type $\mathcal{L}(\theta)$ is to construct a 3/4-ideal tetrahedron out of ideal tetrahedra.

Such a construction can also be used to recast some of the above mentioned formulas for the volume of a finite hyperbolic tetrahedron as decompositions into ideal tetrahedra. This can be helpful in solving scissors congruence problems in hyperbolic space, i.e., problems which ask whether two hyperbolic polyhedra of equal volume are equidecomposable. To see why this is, we consider a case where two polyhedra $A$ and $B$ have the 


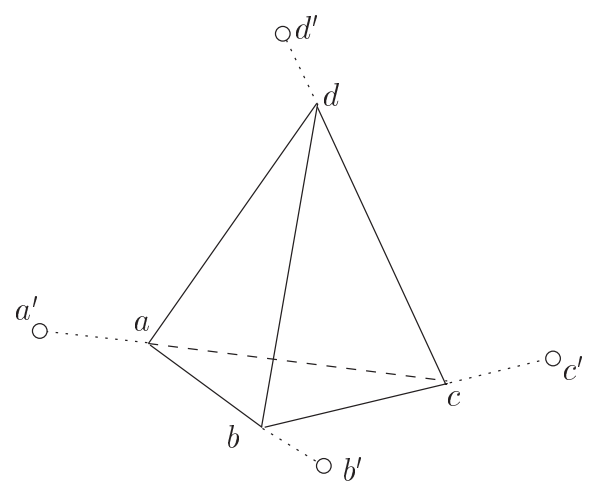

Fig. 2. Constructing a finite tetrahedron out of an ideal tetrahedron and 3/4-ideal tetrahedra: $\{a, b, c, d\}=$ $\left\{a^{\prime}, b^{\prime}, c^{\prime}, d^{\prime}\right\}-\left\{b^{\prime}, d^{\prime}, c^{\prime}, b\right\}-\left\{d^{\prime}, a^{\prime}, c^{\prime}, c\right\}-\left\{a^{\prime}, d^{\prime}, b^{\prime}, a\right\}-\left\{a, b, d, d^{\prime}\right\}$.

same volume. Suppose further that this volume can be written in terms of a formula that simultaneously acts as a decomposition into ideal simplices. It follows that $A$ and $B$ can both be dissected into the same set of tetrahedra. Furthermore, if the volume formula is based on a geometric construction (such as, for example, formulas in [2] and [6]), it is possible to see how these ideal tetrahedra fit together to make $A$ and $B$. A constructive proof that $A$ is scissors congruent to $B$ immediately follows.

The Euclidean version of the question described in the previous paragraph is known as Hilbert's third problem, and was originally posed at the 1900 Congress. Hilbert had asked whether two Euclidean polyhedra with equal volumes are scissors congruent. The negative answer, as well as the counterexample in the form of a cube and a regular tetradron with equal volumes, was quickly provided by Dehn. Dehn also found an invariant necessary for scissors congruence in Euclidean 3-space, now known as the Dehn invariant. It was not until the 1960s that Sydler proved that the Dehn invariant, along with the volume, were, in fact, sufficient for scissors congruence in Euclidean space. Sydler's proof was not constructive, and to this date there exists no algorithm for proving that two polyhedra with equal volume and Dehn invariant are scissors congruent by producing an actual decomposition. Whether Sydler's sufficiency result applies to hyperbolic polyhedra remains an open conjecture. More details on the history and present state of Hilbert's third problem can be found in [4] or [9].

The possibility of constructing a 3/4-ideal tetrahedron out of ideal tetrahedra was first proved in [10], and later in [3]. The former result contains some elements of the results presented here, while the latter one uses purely algebraic methods which offer no obvious geometric clues as to how to perform the actual construction.

In this paper we demonstrate this construction using 10 ideal tetrahedra. The construction consists of three steps. First, we express the 3/4-ideal tetrahedron in terms of three "isosceles" (bilaterally symmetric) 3/4-ideal tetrahedra and one ideal tetrahedron. Next, we express each of the "isosceles" 3/4-ideal tetrahedra in terms of a "supplementary" 3/4-ideal tetrahedron (two of the edges meeting at the finite vertex have supplementary dihedral angles) and an ideal tetrahedron. Finally, we use a construction based on another result in [3] to cut and paste two ideal tetrahedra to make a "supplementary" 3/4-ideal tetrahedron. 


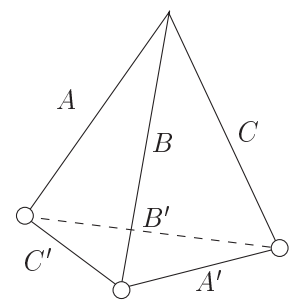

Fig. 3. A 3/4-ideal hyperbolic tetrahedron $T_{3}(A, B, C)$ with dihedral angles $A, B, C$ at the edges meeting at its finite vertex.

\section{Constructing a 3/4-Ideal Tetrahedron out of Isosceles 3/4-Ideal Tetrahedra and Ideal Tetrahedra}

A 3/4-ideal tetrahedron can be determined, up to isometry, by the three dihedral angles meeting at its finite vertex. We denote such a tetrahedron as $T=T_{3}(A, B, C)$, as shown in Fig. 3. We now drop a perpendicular geodesic segment from the non-ideal vertex of $T$ to its ideal face. We denote the endpoint of this geodesic as $O^{\prime}$. The convex hull of the vertices of $T$ and $O^{\prime}$ is shown in Fig. 4.

Lemma 1. Each of the tetrahedra $\left\{r^{\prime}, O^{\prime}, q^{\prime}, v\right\},\left\{p^{\prime}, O^{\prime}, r^{\prime}, v\right\}$, and $\left\{q^{\prime}, O^{\prime}, p^{\prime}, v\right\}$ in Fig. 4 is bilaterally symmetric.

Proof. Consider the polyhedra in Fig. 4 viewed in the half-space model, so that the geodesic segment determined by $v$ and $O^{\prime}$ is normal to the plane at infinity. The projection of the polyhedron $\left\{p^{\prime}, q^{\prime}, r^{\prime}, v, O^{\prime}\right\}$ onto the plane at infinity is shown in Fig. 5. The only way $\left\{v, O^{\prime}\right\}$ can be normal to both the plane at infinity and the plane determined by $\left\{p^{\prime}, q^{\prime}, r^{\prime}\right\}$ is for $O^{\prime}$ to be the circumcenter of the Euclidean triangle $\left\{p^{\prime}, q^{\prime}, r^{\prime}\right\}$. Let $m$ be the midpoint of the projection of $\left\{r^{\prime}, q^{\prime}\right\}$. It then follows by symmetry about the plane

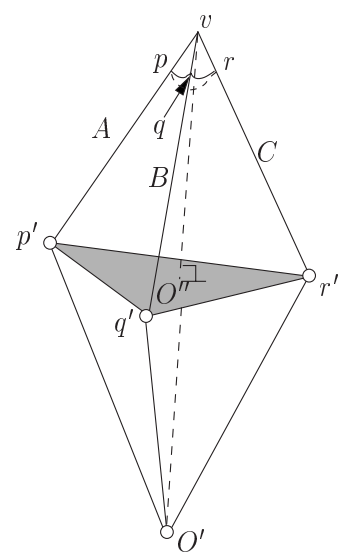

Fig. 4. Subdivision of $T_{3}(A, B, C)$ into three isosceles 3/4-ideal tetrahedra and one ideal tetrahedron. 


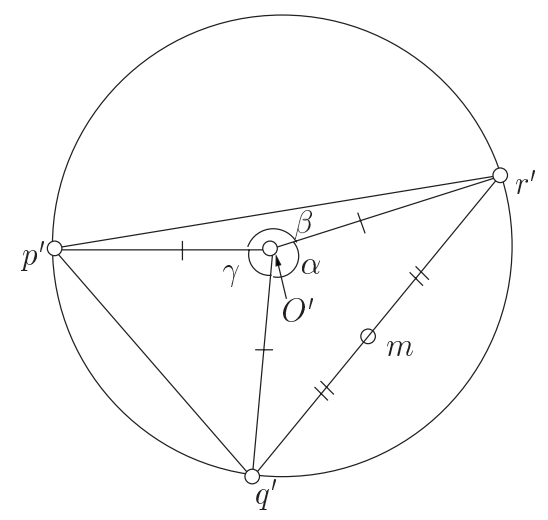

Fig. 5. A projection of Fig. 4 onto the plane at infinity in the half-space model.

determined by $\left\{v, O^{\prime}, m\right\}$ that the dihedral angles of $\left\{r^{\prime}, O^{\prime}, q^{\prime}, v\right\}$ at $\left\{v, r^{\prime}\right\}$ and $\left\{v, q^{\prime}\right\}$ are equal. Analogous results follow for $\left\{q^{\prime}, O^{\prime}, p^{\prime}, v\right\}$ and $\left\{p^{\prime}, O^{\prime}, r^{\prime}, v\right\}$.

We henceforth refer to a bilaterally symmetric 3/4-ideal tetrahedron as an isosceles 3/4-ideal tetrahedron. At this point we can easily determine the dihedral angles of the ideal tetrahedron $\left\{O^{\prime}, p^{\prime}, q^{\prime}, r^{\prime}\right\}$ in terms of the dihedral angles of the three isosceles tetrahedra $\left\{r^{\prime}, O^{\prime}, q^{\prime}, v\right\},\left\{q^{\prime}, O^{\prime}, p^{\prime}, v\right\}$, and $\left\{p^{\prime}, O^{\prime}, r^{\prime}, v\right\}$ at the edge $\left\{v, O^{\prime}\right\}$. We denote these angles as $\alpha, \beta$, and $\gamma$, respectively.

Recall that in Fig. 5 one of the endpoints of $\left\{v, O^{\prime}\right\}$ is the point at infinity, which we denote as $\infty$. Then $\left\{r^{\prime}, O^{\prime}, q^{\prime}, \infty\right\}$ is an ideal tetrahedron whose dihedral angle at $\left\{r^{\prime}, q^{\prime}\right\}$ is $\alpha$ since the edge $\left\{r^{\prime}, q^{\prime}\right\}$ is opposite to the edge $\left\{O^{\prime}, \infty\right\}$. However, the plane determined by $\left\{r^{\prime}, p^{\prime}, q^{\prime}\right\}$ cuts the tetrahedron $\left\{r^{\prime}, O^{\prime}, q^{\prime}, \infty\right\}$ into two congruent parts, since $\infty$ is the image of $O^{\prime}$ under reflection in this plane. Thus, the dihedral angles of $\left\{r^{\prime}, O^{\prime}, q^{\prime}, O^{\prime \prime}\right\}$ and $\left\{r^{\prime}, O^{\prime \prime}, q^{\prime}, \infty\right\}$ at the edge $\left\{r^{\prime}, q^{\prime}\right\}$ (see Fig. 4) are both $\alpha / 2$. Therefore, the dihedral angle of $\left\{O^{\prime}, p^{\prime}, q^{\prime}, r^{\prime}\right\}$ at $\left\{r^{\prime}, q^{\prime}\right\}$ is also $\alpha / 2$. It follows that the other two dihedral angles of $\left\{O^{\prime}, p^{\prime}, q^{\prime}, r^{\prime}\right\}$ are $\beta / 2$ and $\gamma / 2$.

Remark 1. The construction described above can be viewed as a generalization of Milnor's decomposition of an ideal tetrahedron into three isosceles 3/4-ideal tetrahedra in [5]. As $v$ tends to $\infty$, the polyhedron in Fig. 4 becomes Milnor's construction.

In order to determine the dihedral angles of $\left\{r^{\prime}, O^{\prime}, q^{\prime}, v\right\},\left\{p^{\prime}, O^{\prime}, r^{\prime}, v\right\}$, and $\left\{q^{\prime}, O^{\prime}\right.$, $\left.p^{\prime}, v\right\}$ we use a bit of spherical trigonometry. We place a small sphere $S$ centered at $v$ and look at the spherical triangle formed by the intersection of $T$ with $S, \triangle p q r$ in Fig. 4. The angles of $\triangle p q r$ are the same as the dihedral angles of $T$. Each side length of $\triangle p q r$ is equal to $\rho \theta$, where $\rho$ is the radius of $S$ and $\theta$ is the angle at $v$ of the corresponding face of $T$. It is convenient to scale $S$ so that $\rho=1$. By abuse of notation, we refer to the scaled version of $\triangle p q r$ as $\triangle p q r$. Then the side lengths of $\triangle p q r$ are exactly the angles of the faces of $T$. 


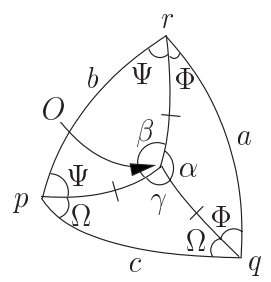

Fig. 6. Subdivision of an arbitrary spherical triangle into three isosceles spherical triangles.

Let $O$ be the intersection of $\left\{v, O^{\prime}\right\}$ with $\triangle p q r$. It follows that $\triangle q r O, \triangle r p O$, and $\triangle p q O$ have the same angles as the dihedral angles of $\left\{r^{\prime}, O^{\prime}, q^{\prime}, v\right\},\left\{p^{\prime}, O^{\prime}, r^{\prime}, v\right\}$, and $\left\{q^{\prime}, O^{\prime}, p^{\prime}, v\right\}$ at the edges meeting at $v$. In particular, $\triangle q r O, \triangle r p O$, and $\triangle p q O$ are isosceles. It remains to find the six angles denoted by Greek letters in Fig. 6. The angles $\Phi, \Psi$, and $\Omega$ can be easily determined by solving the system of equations

$$
\begin{aligned}
& \Omega+\Psi=A, \\
& \Omega+\Phi=B, \\
& \Psi+\Phi=C,
\end{aligned}
$$

where $A, B$, and $C$ are angles of the original spherical triangle. Thus

$$
\Phi=\frac{B+C-A}{2}, \quad \Psi=\frac{A+C-B}{2}, \quad \Omega=\frac{A+B-C}{2} .
$$

To determine the angles $\alpha, \beta$, and $\gamma$ we use the spherical law of cosines,

$$
\cos C=-\cos A \cos B+\sin A \sin B \cos C,
$$

where the capital letters denote the angles of a spherical triangle and the lowercase letters denote the corresponding side. First we use (4) to obtain the expressions for the side lengths $a, b$, and $c$ in Fig. 6 in terms of the dihedral angles of $T$ (we assume that $\triangle p q r$ lives on a sphere of radius 1$)$ :

$$
\begin{aligned}
& \cos a=\frac{\cos A+\cos B \cos C}{\sin B \sin C}, \\
& \cos b=\frac{\cos B+\cos A \cos C}{\sin A \sin C}, \\
& \cos c=\frac{\cos C+\cos A \cos B}{\sin A \sin B} .
\end{aligned}
$$

Then we use (4) again to find

$$
\alpha=\arccos \left(-\cos ^{2} \Phi+\sin ^{2} \Phi \cos a\right),
$$




$$
\begin{aligned}
& \beta=\arccos \left(-\cos ^{2} \Psi+\sin ^{2} \Psi \cos b\right), \\
& \gamma=\arccos \left(-\cos ^{2} \Omega+\sin ^{2} \Omega \cos c\right),
\end{aligned}
$$

where $\Phi, \Psi$, and $\Omega$ are given in (3).

To summarize the results obtained in this section, we have found that the expression relating the tetrahedra in Fig. 4,

$$
\left\{p^{\prime}, q^{\prime}, r^{\prime}, v\right\}=\left\{r^{\prime}, O^{\prime}, q^{\prime}, v\right\}+\left\{p^{\prime}, O^{\prime}, r^{\prime}, v\right\}+\left\{q^{\prime}, O^{\prime}, p^{\prime}, v\right\}-\left\{p^{\prime}, r^{\prime}, q^{\prime}, O^{\prime}\right\},
$$

leads to the identity

$$
T_{3}(A, B, C)=T_{3}(\alpha, \Phi, \Phi)+T_{3}(\beta, \Psi, \Psi)+T_{3}(\gamma, \Omega, \Omega)-T(\alpha / 2, \beta / 2, \gamma / 2),
$$

where $\alpha, \beta, \gamma, \Phi, \Psi$, and $\Omega$ are given in (3), (5), and (6), and $T(\alpha / 2, \beta / 2, \gamma / 2)$ represents an ideal tetrahedron with dihedral angles $\alpha / 2, \beta / 2, \gamma / 2$ at each of its vertices.

\section{Constructing an Isosceles 3/4-Ideal Tetrahedron out of a "Supplementary" 3/4-Ideal Tetrahedron and an Ideal Tetrahedron}

We begin with the following

Definition 1. A supplementary 3/4-ideal tetrahedron is a 3/4-ideal tetrahedron having the property that two of the dihedral angles at the edges meeting at its finite vertex are supplementary.

We are interested in supplementary 3/4-ideal tetrahedra because any such tetrahedron can be constructed out of two ideal tetrahedra, as will be seen in Section 4. We now show how to express an isosceles 3/4-ideal tetrahedron in terms of an ideal tetrahedron and a supplementary 3/4-ideal tetrahedron.

Consider the isosceles 3/4-ideal tetrahedron $\left\{r^{\prime}, O^{\prime}, q^{\prime}, v\right\}$ in Fig. 4. Now extend the finite end of the ray $\left\{r^{\prime}, v\right\}$ to infinity and call this endpoint $v_{r}^{\infty}$ (by symmetry, $\left\{q^{\prime}, v\right\}$ works equally well). Connect $v_{r}^{\infty}$ to $O^{\prime}$ and $q^{\prime}$ by geodesic segments as shown in Fig. 7. We claim that the tetrahedron $\left\{q^{\prime}, O^{\prime}, v_{r}^{\infty}, v\right\}$ is supplementary. Recall that the dihedral angles of $\left\{r^{\prime}, O^{\prime}, q^{\prime}, v\right\}$ at the edges $\left\{v, r^{\prime}\right\},\left\{v, q^{\prime}\right\}$, and $\left\{v, O^{\prime}\right\}$ are $\Phi, \Phi$, and $\alpha$, respectively. It then follows easily from Fig. 4 that the dihedral angles of $\left\{q^{\prime}, O^{\prime}, v_{r}^{\infty}, v\right\}$ at the edges $\left\{v, v_{r}^{\infty}\right\},\left\{v, q^{\prime}\right\}$, and $\left\{v, O^{\prime}\right\}$ are $\Phi, \pi-\Phi$, and $\pi-\alpha$. Thus $\left\{q^{\prime}, O^{\prime}, v_{r}^{\infty}, v\right\}$ is a supplementary 3/4-ideal tetrahedron.

The dihedral angles of the ideal tetrahedron $\left\{r^{\prime}, O^{\prime}, q^{\prime}, v_{r}^{\infty}\right\}$ are the same as those of $\left\{r^{\prime}, O^{\prime}, q^{\prime}, v\right\}$ at the vertex $r^{\prime}$, namely $(\pi+\alpha) / 2-\Phi,(\pi-\alpha) / 2$, and $\Phi$. Therefore, we can express the decomposition

$$
\left\{r^{\prime}, O^{\prime}, q^{\prime}, v\right\}=\left\{r^{\prime}, O^{\prime}, q^{\prime}, v_{r}^{\infty}\right\}-\left\{q^{\prime}, O^{\prime}, v_{r}^{\infty}, v\right\}
$$

as

$$
T_{3}(\alpha, \Phi, \Phi)=T\left(\frac{\pi+\alpha}{2}-\Phi, \frac{\pi-\alpha}{2}, \Phi\right)-T_{3}(\pi-\alpha, \Phi, \pi-\Phi)
$$




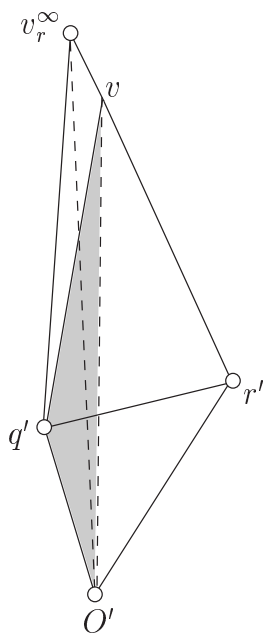

Fig. 7. Decomposition of a supplementary $3 / 4$-ideal tetrahedron into an ideal tetrahedron and an isosceles 3/4-ideal tetrahedron.

Similarly, we obtain

$$
T_{3}(\beta, \Psi, \Psi)=T\left(\frac{\pi+\beta}{2}-\Psi, \frac{\pi-\beta}{2}, \Psi\right)-T_{3}(\pi-\beta, \Psi, \pi-\Psi)
$$

and

$$
T_{3}(\gamma, \Omega, \Omega)=T\left(\frac{\pi+\gamma}{2}-\Omega, \frac{\pi-\gamma}{2}, \Omega\right)-T_{3}(\pi-\gamma, \Omega, \pi-\Omega) .
$$

\section{Constructing a Supplementary 3/4-Ideal Tetrahedron out of Two Ideal Tetrahedra}

We begin by introducing some notation. Consider an ideal tetrahedron in the half-space model, and identify the plane at infinity with the extended complex plane $\mathbb{C} \cup\{\infty\}$. Then this tetrahedron can be determined up to orientation by the cross ratio of the complex coordinates of its vertices, which is defined as follows:

Definition 2. Let $a, b, c, d \in \mathbb{C} \cup \infty$. Then the cross ratio of $a, b, c, d$, denoted as $(a, b, c, d)$, is defined as $(a-c)(b-d) /(a-d)(b-c)$.

The details of this notation can be found in [9].

Following the convention in [3], we denote the ideal tetrahedron with vertices $\{\infty$ : $0: 1: z\}$ whose projection onto the plane at infinity is shown in Fig. 8 as $\{z\}$.

The idea for the construction presented in this section comes from Dupont and Sah's proof that the group of polyhedra generated by cross ratios is 2-divisible, i.e.,

$$
\left\{z^{2}\right\}=2\{z\}+2\{-z\} .
$$




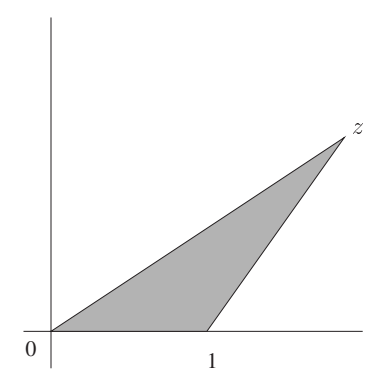

Fig. 8. Projection onto the half-plane of an ideal hyperbolic tetrahedron with cross ratio $z$.

Since any ideal hyperbolic tetrahedron can be represented by $\left\{z^{2}\right\}$ for some $z \in \mathbb{C}$, (12) says that any ideal simplex can be divided into two scissors congruent parts, each of which is comprised of $\{z\}$ and $\{-z\}$. We now show how to cut and paste $\{z\}$ and $\{-z\}$ into a supplementary 3/4-ideal tetrahedron.

First, consider the geometric proof of identity (12) as shown in Fig. 9. In that figure, $\left\{\infty: 0: 1: z^{2}\right\}=\left\{z^{2}\right\}$. The other four tetrahedra in the figure are $\{\infty: 0: 1: z\}=\{z\}$, $\left\{\infty: 0: z: z^{2}\right\}=\{z\},\left\{1: z^{2}: z: 0\right\}=\{-z\}$, and $\left\{z: \infty: z^{2}: 1\right\}=\{-z\}$. Thus, the identity (12) can be read off Fig. 9.

Now, imagine that the point $p$ is on the geodesic connecting the points 1 and $z^{2}$, so that in Fig. 9 we are seeing its projection onto the plane at infinity.

Lemma 2. The tetrahedron $\{0,1, p, \infty\}$ is scissors congruent to the tetrahedron $\{0,1, z, \infty\}$ with cross ratio $\{z\}$ minus the tetrahedron $\left\{1, z, z^{2}, \infty\right\}$ with cross ratio $-\{-z\}$.

Proof. Consider the isometry $\varphi: \mathbb{C} \cup\{\infty\} \longrightarrow \mathbb{C} \cup\{\infty\}$ defined by

$$
\varphi: \omega \mapsto z^{2} / \omega .
$$

We claim that $\varphi$ fixes the point $p$. To see why this is, note that $\varphi$ interchanges 0 and $\infty$ while fixing $z$. Therefore, the plane determined by $0, \infty$, and $z$ remains unchanged under the action of $\varphi$. Since the point $p$ lies in that plane, as well as on the geodesic $\left\{1, z^{2}\right\}$ also fixed by $\varphi$, we have that $\varphi(p)=p$.

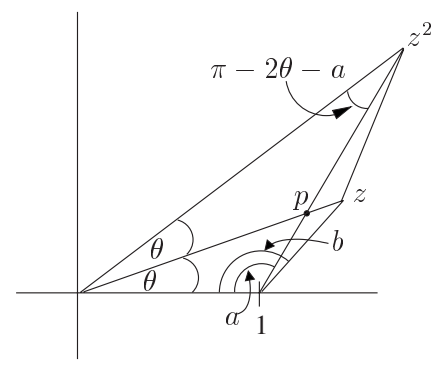

Fig. 9. Geometric proof of the identity $\left\{z^{2}\right\}=2\{z\}+2\{-z\}$. 
It follows that $\left\{p, z, z^{2}, \infty\right\}$ is congruent to $\{p, z, 1,0\}$ by the action of $\varphi$. Therefore, half of $\left\{z^{2}\right\}$ is

$$
\begin{aligned}
\{0,1, z, \infty\}-\left\{1, z, z^{2}, \infty\right\} & =\{0,1, z, \infty\}-\{1, z, p, \infty\}-\left\{p, z, z^{2}, \infty\right\} \\
& =\{0,1, z, \infty\}-\{1, z, p, \infty\}-\{p, z, 1,0\} \\
& =\{0,1, p, \infty\}
\end{aligned}
$$

It turns out that the tetrahedra $\{0,1, p, \infty\}$ and $\left\{0, p, z^{2}, \infty\right\}$ are not only scissors congruent, but also congruent. Since a $3 / 4$-ideal tetrahedron is determined by the dihedral angles at its non-ideal vertex, it is sufficient to consider the angles at the vertex $p$. In Fig. 9 the dihedral angle at the edge $\left\{1, z^{2}\right\}$ is $2 \theta$ for both $\{0,1, p, \infty\}$ and $\left\{0, p, z^{2}, \infty\right\}$, since that angle is opposite the angle at $\{0, \infty\}$ in the ideal tetrahedron $\left\{0,1, z^{2}, \infty\right\}$. It can be easily seen from Fig. 9 that the dihedral angles of $\{0,1, p, \infty\}$ and $\left\{0, p, z^{2}, \infty\right\}$ at the edge $\{p, \infty\}$ are $\pi-\theta-a$ and $\theta+a$. Using the fact that the dihedral angles of a tetrahedron at an ideal vertex add up to $\pi$, we find that the dihedral angles of $\{0,1, p, \infty\}$ and $\left\{0, p, z^{2}, \infty\right\}$ at the edge $\{0, p\}$ are $\theta+a$ and $\pi-\theta-a$. Since the $3 / 4$-ideal tetrahedra $\{0,1, p, \infty\}$ and $\left\{0, p, z^{2}, \infty\right\}$ each have angles $2 \theta, \theta+a, \pi-\theta-a$ at their finite vertex, they are congruent to each other.

\section{Putting All the Pieces Together}

All that is left now is to express each supplementary 3/4-ideal tetrahedron on the righthand side of (9)-(11) in terms of the two ideal tetrahedra that are scissors congruent to it. That is, given a supplementary $3 / 4$-ideal tetrahedron $T_{3}(\xi, w, \pi-w)$ we need to find the corresponding $\theta, a$, and $b$ in Fig. 9. We can think of $\theta, a$, and $b$ as being real-valued functions of the dihedral angles of the given supplementary 3/4-ideal tetrahedron. Then

$$
\theta(\xi, w)=\xi / 2
$$

and

$$
a(\xi, w)=w-\xi / 2 .
$$

Then the Euclidean law of sines gives

$$
\left|z^{2}\right|=\frac{\sin a(\xi, w)}{\sin (2 \theta(\xi, w)+a(\xi, w))} .
$$

It follows that

$$
\begin{aligned}
b(\xi, w) & =\pi-\arg (z-1) \\
& =\pi-\arg \left(\sqrt{\left|z^{2}\right|} e^{i \theta(\xi, w)}-1\right) \\
& =\pi-\arctan \left(\frac{\sqrt{\sin a(\xi, w)} \sin \theta(\xi, w)}{\sqrt{\sin a(\xi, w)} \cos \theta(\xi, w)-\sqrt{\sin (2 \theta(\xi, w)+a(\xi, w))}}\right) .
\end{aligned}
$$


Therefore, $\{z\}$ in Fig. 9 corresponds to $T(\theta(\xi, w), b(\xi, w), \pi-\theta(\xi, w)-b(\xi, w))$ and $-\{-z\}$ corresponds to $T(\pi-\theta(\xi, w), b(\xi, w)-a(\xi, w), \theta(\xi, w)-b(\xi, w)+a(\xi, w))$. It follows that

$$
\begin{aligned}
T(\xi, w, \pi-w)= & T(\theta(\xi, w), b(\xi, w), \pi-\theta(\xi, w)-b(\xi, w)) \\
& -T(\pi-\theta(\xi, w), b(\xi, w)-a(\xi, w), \theta(\xi, w) \\
& -b(\xi, w)+a(\xi, w)) .
\end{aligned}
$$

Putting together (8)-(11) and (18) gives us the following breakdown of an arbitrary 3/4-ideal tetrahedron into ideal tetrahedra:

$$
\begin{aligned}
T_{3}(A, B, C)= & T\left(\frac{\pi+\alpha}{2}-\Phi, \frac{\pi-\alpha}{2}, \Phi\right)+T\left(\frac{\pi+\beta}{2}-\Psi, \frac{\pi-\beta}{2}, \Psi\right) \\
+ & T\left(\frac{\pi+\gamma}{2}-\Omega, \frac{\pi-\gamma}{2}, \Omega\right)-T\left(\frac{\alpha}{2}, \frac{\beta}{2}, \frac{\gamma}{2}\right) \\
& -T(\theta(\pi-\alpha, \Phi), b(\pi-\alpha, \Phi), \pi-\theta(\pi-\alpha, \Phi)-b(\pi-\alpha, \Phi)) \\
+ & T(\pi-\theta(\pi-\alpha, \Phi), b(\pi-\alpha, \Phi)-a(\pi-\alpha, \Phi), \\
& \quad \theta(\pi-\alpha, \Phi)-b(\pi-\alpha, \Phi)+a(\pi-\alpha, \Phi)) \\
- & T(\theta(\pi-\beta, \Psi), b(\pi-\beta, \Psi), \pi-\theta(\pi-\beta, \Psi) \\
& -b(\pi-\beta, \Psi)) \\
+ & T(\pi-\theta(\pi-\beta, \Psi), b(\pi-\beta, \Psi)-a(\pi-\beta, \Psi), \\
& \theta(\pi-\beta, \Psi)-b(\pi-\beta, \Psi)+a(\pi-\beta, \Psi)) \\
- & T(\theta(\pi-\gamma, \Omega), b(\pi-\gamma, \Omega), \pi-\theta(\pi-\gamma, \Omega)-b(\pi-\gamma, \Omega)) \\
+ & T(\pi-\theta(\pi-\gamma, \Omega), b(\pi-\gamma, \Omega)-a(\pi-\gamma, \Omega), \\
& \quad \theta(\pi-\gamma, \Omega)-b(\pi-\gamma, \Omega)+a(\pi-\gamma, \Omega)),
\end{aligned}
$$

where $A, B, C$ are arbitrary, $\alpha, \beta, \gamma$ are given in (6), $\Phi, \Psi$, and $\Omega$ are given in (3), and $a, b$, and $\theta$ are defined by (15), (17), and (14).

\section{Conclusion}

A 3/4-ideal tetrahedron is closely related to a tetrahedron with three ideal vertices and one hyperideal vertex, as described in Section 2 of [7]. In fact the volume formula (2) applies equally well to such a tetrahedron, which can also be viewed as one of the symmetric halves of an ideal triangular prism. However, the construction described in this paper breaks down when it comes to a hyperideal tetrahedron. For one, the sum of the dihedral angles at a hyperideal vertex is less than $\pi$, so that the whole notion of a supplementary 3/4-ideal tetrahedron no longer makes sense when the finite vertex becomes hyperideal. It would be interesting to come up with a construction of a simplex with a hyperideal vertex out of ideal simplices, and to see whether it has anything in common with the construction described above. 


\section{Acknowledgements}

I express my sincerest thanks to Justin Roberts for encouraging me to pursue the ideas in Section 4 that ultimately led to this proof. I also thank and acknowledge Peter Doyle, who showed me how to reduce the number of tetrahedra in the original construction.

\section{References}

1. Y. Cho and H. Kim, On the volume formula for hyperbolic tetrahedra, Discrete Comput. Geom. 22 (1999), 347-366.

2. P. Doyle and G. Leibon, 23040 symmetries of hyperbolic tetrahedra, arXiv: math GT/0309187.

3. J. L. Dupont and C.-H. Sah, Scissors congruences, II, J. Pure Appl. Algebra 25 (1982), 159-195.

4. R. Kellerhals, Old and new about Hilbert's third problem, in European Women in Mathematics (Loccum, 1999), pp. 179-187, Hindawi, Cairo, 2000.

5. J. Milnor, Hyperbolic geometry: the first 150 years, Bull. Amer. Math. Soc. 6 (1982), 9-24.

6. Y. Mohanty, Hyperbolic Polyhedra: Volume and Scissors Congruence, Ph.D. thesis, University of California, San Diego, La Jolla, CA, 2002.

7. Y. Mohanty, The Regge symmetry is a scissors congruence in hyperbolic space, Algebra Geom. Topol. 3 (2003), 1-31.

8. J. Murakami and M. Yano, On the volume of a hyperbolic and spherical tetrahedron, Preprint (2002).

9. W. D. Neumann, Hilbert's 3rd problem and invariants of 3-manifolds, Geom. Topol. Monogr. 1 (1998), 383-411.

10. C.-H. Sah, Scissors congruences, I, Gauss-Bonnet map, Math. Scand. 49 (1982), 181-210.

11. E. B. Vinberg, Volumes of non-Euclidean polyhedra, Russian Math. Surveys 48(2) (1993), 15-45.

Received January 4, 2003, and in revised form April 30, 2003. Online publication February 9, 2004. 